\title{
Prostheses and Exoskeletons: Moving From a Mechatronics Bottleneck through a Controls Bottleneck
}

\author{
Jon Sensinger \\ Institute of Biomedical Engineering / University of New Brunswick \\ 25 Dineen Dr, Fredericton, Canada \\ sensinger@ieee.org
}

\section{Extended Abstract}

The human body is an amazing design that is in many ways superior to anthropometric robots, whether throwing a ball [1] or tying a knot. This contrast becomes clear when the person becomes injured and we attempt to augment it with a robotic interface such as a prosthetic limb or a powered exoskeleton to regain mobility. Existing robotic augmentation systems tend to be heavy and have significant inertias due to high transmission ratios [2]. But equally important, anthropometric robots tend to have difficulty rendering accurate forces (again due to their high gear ratios) or achieve stable gait in the presence of environmental perturbations, and these control challenges are amplified when the robot must coordinate activities with the user. This is a complex problem that requires an accurate understanding of interaction between the mechatronic system, the control platform, and the human.

Recent advances by ourselves [3]-[7] and others [8]-[10] have enabled us to transition from a mechatronic bottleneck to a control bottleneck in powered prostheses and exoskeletons. This is an exciting time in the field in which a variety of mechatronic platforms are available to answer a host of clinically relevant controls problems, with an ultimate goal to improve the lives of people who have a disability. This abstract covers our recent work in upper and lower limb control interfaces.

Upper-limb control interface. In the field of upper-limb prostheses we have developed a principled approach that broadly falls within the realm of a nonlinear quadratic Gaussian controller [11] to understand how the user controls a device in light of its noise (myoelectric control signals have substantial multiplicative sensor noise) and reduced feedback (many of the sensors in your arm are lost in amputation). Our modelling approach is able to accurately predict the ways that people compensate for reduced control certainty and reduced visual feedback [12]-[14]. Using essentially an inverse-model approach, we are now predicting optimal control and sensory feedback dynamics in order to minimize uncertainty and maximize performance in light of the user's known control strategies.

Lower-limb control interface. Using nonlinear control approaches, we have taken what is typically a nonautonomous trajectory-defined problem and turned it into an autonomous phase-defined problem through the use of virtual constraints [15]. This has enabled us to achieve stable, robust walking of robotic prosthetic legs that we are currently extending to exoskeleton control. Our approach has been shown to match human-like adaptation [16], demonstrated to be theoretically stable without an accurate model of the user or prosthesis [17], [18]; demonstrated robustness in lab-testing even in the presence of speed perturbations [19], and is appreciated by clinicians and patients. We are currently extending this approach to hip control in an exoskeleton.

With the recent development of appropriate hardware, control problems are starting to be addressed at a level that can truly impact patient care within the field of rehabilitation engineering. The fusion of new mechatronic technologies with advances in control theory should result in tangible impacts in clinical care within the next decade.

\section{References}

[1] A. Albu-Schaffer, O. Eiberger, M. Grebenstein, S. Haddadin, C. Ott, T. Wimbock, S. Wolf, and G. Hirzinger, "Soft robotics - From torque feedback-controlled lightweight robots to intrinsically compliant systems," IEEE Robot. Autom. Mag., vol. 15, no. 3, pp. 20-30, 2008.

[2] R. F. ff. Weir and J. W. Sensinger, "Design of Artificial Arms and Hands for Prosthetic Applications," in Biomedical Engineering and Design Handbook, 2nd ed., vol. 2, M. Kutz, Ed. New York: McGraw-Hill, 2009, pp. 537-598. 
[3] J. W. Sensinger, "Selecting motors for robots using biomimetic trajectories: optimum benchmarks, windings, and other considerations," in IEEE Conference on Robotics and Automation. Anchorage, Alaska, pp. 4175-4181, 2010.

[4] J. W. Sensinger, S. D. Clark, and J. F. Schorsch, "Exterior vs. Interior Rotors in Robotic Brushless Motors," in IEEE Conference on Robotics and Automation. Shanghai, China, pp. 2764-2770, 2011.

[5] J. W. Sensinger and J. H. Lipsey, "Cycloid vs. Harmonic Drives for use in High Ratio, Single Stage Robotic Transmissions," in IEEE Conference on Robotics and Automation, 2012, pp. 4130-4135.

[6] J. W. Sensinger, "Unified approach to cycloid drive profile, stress, and efficiency optimization," ASME J. Mech. Des., vol. 132, no. 2, pp. 1-5, 2010.

[7] J. W. Sensinger, "Efficiency of high-sensitivity gear trains, such as cycloid drives," ASME J. Mech. Des., vol. 135, no. 7, 2013.

[8] F. Sup, H. A. Varol, J. Mitchell, T. J. Withrow, and M. Goldfarb, "Preliminary Evaluations of a Self-Contained Anthropomorphic Transfemoral Prosthesis.," IEEE ASME Trans. Mechatron., vol. 14, no. 6, pp. 667-676, Jan. 2009.

[9] J. A. Blaya and H. Herr, "Adaptive control of a variable-impedance ankle-foot orthosis to assist drop-foot gait," IEEE Trans. Neural Syst. Rehabil. Eng., vol. 12, no. 1, pp. 24-31, 2004.

[10] J. K. Hitt, T. G. Sugar, M. Holgate, and R. Bellman, "An Active Foot-Ankle Prosthesis With Biomechanical Energy Regeneration," J. Med. Devices-Transactions ASME, vol. 4, no. 1, 2010.

[11] W. Li and E. Todorov, "Iterative linearization methods for approximately optimal control and estimation of non-linear stochastic system," Int. J. Control, vol. 80, no. 9, pp. 1439-1453, Sep. 2007.

[12] J. W. Sensinger, A. Aleman-Zapata, and K. Englehart, "Do cost functions for tracking error generalize across tasks with different noise levels?," PLoS One, vol. 10, no. 8, p. e0136251, 2015.

[13] R. E. Johnson, K. P. Kording, L. J. Hargrove, and J. W. Sensinger, "EMG versus torque control of human-machine systems: equalizing control signal variability does not equalize error or uncertainty," Ieee Trans. Neural Syst. Rehabil. Eng., vol. Submitted, 2016.

[14] R. E. Johnson, K. P. Kording, L. J. Hargrove, and J. W. Sensinger, "Adapting to random and systematic errors: How amputees using myoelectric control differ from intact-limbed subjects using reaching movements," J. Neural Eng., vol. Submitted, 2016.

[15] E. R. Westervelt, J. W. Grizzle, C. Chevallereau, J. H. Choi, and B. Morris, Feedback Control of Dynamic Bipedal Robot Locomotion. New York, NY: CRC Press, 2007.

[16] R. D. Gregg, E. Rouse, L. J. Hargrove, and J. Sensinger, "Evidence for a Time-Invariant Phase Variable in Human Ankle Control," PLoS One, vol. 9, no. 2, p. e89163, 2014.

[17] R. D. Gregg, T. Lenzi, L. Hargrove, and J. Sensinger, "Virtual constraint control of a powered prosthetic leg: from simulation to experiments with transfemoral amputees," IEEE Trans. Robot., vol. 30, no. 6, pp. 1455-1471, 2014.

[18] R. D. Gregg and J. W. Sensinger, “Towards biomimetic virtual constraint control of a powered prosthetic leg," IEEE Trans. Control Syst. Technol., vol. 22, no. 1, pp. 246-254, 2014.

[19] T. Lenzi, L. J. Hargrove, and J. Sensinger, "A novel control framework allowing physiological walking speed adaptation in powered transfemoral prostheses," IEEE Robot. Autom. Mag., vol. 24, no. 4, pp. 94-107, 2014. 